\title{
Identification of a Geranylgeranyl reductase gene for chlorophyll synthesis in rice
}

Pingyu Wang, Chunmei Li, Yang Wang, Rui Huang, Changhui Sun, Zhengjun Xu, Jianqing Zhu, Xiaoling Gao, Xiaojian Deng ${ }^{*}$ and Pingrong Wang ${ }^{*}$

\begin{abstract}
Geranylgeranyl reductase (CHL P) catalyzes the reduction of geranylgeranyl diphosphate to phytyl diphosphate, and provides phytol for both Chlorophyll (Chl) and tocopherol synthesis. In this study, we isolated a yellow-green leaf mutant, 502ys, in rice (Oryza sativa). The mutant exhibited reduced level of Chls, arrested development of chloroplasts, and retarded growth rate. The phenotype of the 502ys mutant was controlled by by a recessive mutation in a nuclear gene on the long arm of rice chromosome 2. Map-based cloning of the mutant resulted in the identification of an OsChI P gene (LOC_Os02g51080). In the 502ys mutant, a single base pair mutation was detected at residue 1279 in DNA sequence of the gene, resulting in an amino acid change (Gly-206 to Ser) in the encoded protein. HPLC analysis of Chls indicated that the majority of $\mathrm{Chl}$ molecules are conjugated with an unsaturated geranylgeraniol side chain, in addition to small amount of normal Chls in the mutant. Furthermore, the mutant phenotype was complemented by transformation with the wild-type gene. Therefore, this study has confirmed the 502ys mutant resulted from a single base pair mutation in OsChl P gene.
\end{abstract}

Keywords: Geranylgeranyl reductase; Chl P gene; Chlorophyll; Rice

\section{Introduction}

Chlorophyll $(\mathrm{Chl})$ is the main component of the photosynthetic pigments. Chl molecules consist of two parts: a hydrophilic tetrapyrrole moiety (chlorophyllide, Chlide) and a hydrophobic alcohol moiety (phytyl, Phy), which are formed from the precursor molecules glutamyltRNA and isopentenyl diphosphate, respectively, in two different pathways of tetrapyrrole and isoprenoid biosynthesis. The tetrapyrrole biosynthesis, entirely locating in plastids, has been extensively studied with various organisms by biochemical and genetic methods. So far, almost all genes for 15 steps of tetrapyrrole biosynthetic pathway have been identified in higher plants represented by Arabidopsis (Beale 2005; Nagata et al. 2005; Wu et al. 2007; Islam et al. 2008; Wang et al. 2010, 2013; Sakuraba et al. 2013).

The branched isoprenoid pathway is rather complex and comprises enzymatic steps in at least two compartments. In plants, Phy represents the side chain of Chls, tocopherols (TP) and phylloquinones, and is necessary

*Correspondence: xjdeng2006@aliyun.com; pingrong_wang@aliyun.com
Rice Research Institute, Sichuan Agricultural University, Chengdu 611130, Sichuan, China for their integration into plastidmembranes (Soll et al. 1980, 1983; Soll 1987; Bollivar et al. 1994). In both Chl and TP synthesis, the Phy chain is provided by geranylgeranyl pyrophosphate (GGPP), a plastidial isoprenoid, formed by four molecules of isopentenyl pyrophosphate (IPP), which are derived from the cytosolic and chloroplastidic pathways (Rohmer et al. 1993; Lichtentaler et al. 1997). In Chl synthesis, GGPP can either be reduced to phytyl pyrophosphate (PhyPP) and esterified with Chlide to generate phytyl $\mathrm{Chl}\left(\mathrm{Chl}_{\mathrm{Phy}}\right)$, or first esterified with Chlide to form geranylgeranylated $\mathrm{Chl}$ $\left(\mathrm{Chl}_{\mathrm{GG}}\right)$ and then stepwise reduced into $\mathrm{Chl}_{\mathrm{Phy}}$ (Soll et al. 1983; Bollivar et al. 1994; Keller et al. 1998; Chew et al. 2008). In the TP pathway, tocopherols are generally believed to arise from the condensation of homogentisic acid and PhyPP (Schultz et al. 1985; Soll et al. 1980; Collakova and DellaPenna 2001; Savidge et al. 2002; Cahoon et al. 2003). Therefore, CHL P is demonstrated to provide Phy for both Chl and TP synthesis.

CHL P catalyzes the reduction of $\mathrm{Chl}_{\mathrm{GG}} a$ into $\mathrm{Chl}_{\mathrm{Phy}}$ $a$ as well as the reduction of free GGPP into PhyPP (Keller et al. 1998). Genes encoding CHL P have been characterized in photosynthetic bacteria such as Syneclrocystis sp.

\section{包 Springer}


PCC6803, Rhodobacter sphaeroides and Chlorobaculum tepidum (Addlesee et al. 1996; Addlesee and Hunter. 1999; Chew et al. 2008), and in higher plants such as Arabidopsis (Arabidopsis thaliana), tobacco (Nicotiana tabacum), peach (Prunus persica), olive (Olea europaea) and sesame (Sesamum indicum) (Keller et al. 1998; Tanaka et al. 1999; Giannino et al. 2004; Bruno et al. 2009; Park et al. 2010). But until recently, no Chl P gene was identified in monocotyledonous plants (Zhou et al. 2013).

In this study, we isolated a rice yellow-green leaf mutant, 502ys, from ethyl methanesulfonate (EMS) mutagenesis. The mutant exhibited reduced level of Chls, arrested development of chloroplasts, and retarded growth rate. Map-based cloning of the mutant resulted in identification of the OsChl P gene. In the $502 y s \mathrm{mu}-$ tant, a single base pair mutation was detected in DNA sequence of the gene, resulting in an amino acid change in the encoded protein. HPLC analysis of Chls indicated that the majority of Chls molecules are conjugated with an unsaturated geranylgeraniol side chain, in addition to small amount of normal Chls in the mutant. Furthermore, the mutant phenotype was complemented by transformation with the wild-type gene. Therefore, this study has confirmed the 502ys mutant resulted from a single base pair mutation in OsChl P gene.

\section{Materials and methods}

\section{Plant materials and mapping population}

The 502ys mutant was a yellow-green leaf mutant isolated from the progeny of japonica cv Nipponbare treated with $0.7 \%$ of ethyl methanesulfonate (v: v, Sigma). The $\mathrm{F}_{2}$ mapping population was generated by crossing the 502ys mutant with normal green indica cV Minghui 63. All rice materials were planted under natural conditions in April to August, in Wenjiang District (Latitude $30^{\circ} 42^{\prime} \mathrm{N}$, Longitude $103^{\circ} 50^{\prime} \mathrm{E}$, and Altitude 539.3 m), Chengdu City, Sichuan, China.

\section{Marker development}

The SSR markers were obtained from Gramene (http:// archive.gramene.org/markers/microsat/) based on the SSR linkage map constructed by McCouch et al. (2002). To narrow down the region of the target locus, the BLAST search in the National Center for Biotechnology Information database was conducted to find insertion/deletion (InDel) sequence divergence between japonica cv Nipponbare and indica cv 9311 around this locus (http://www.ncbi. nlm.nih.gov/BLAST/), and InDel markers were designed around the sequence divergence including 15 to $100 \mathrm{bp}$ InDel using the Primer 5.0 software.

\section{Sequence analysis}

The full-length DNA and protein sequences of OsChl P and its homologs were retrieved from GenBank (http:// www.ncbi.nlm.nih.gov). The chloroplast signal peptide was predicted with http://www.cbs.dtu.dk/services/TargetP (Emanuelsson et al. 2000). Multiple sequence alignment and phylogenetic analysis were conducted using DNAMAN version 6.0 (Lynnon Biosoft).

\section{Transmission electron microscopy analysis}

Rice leaf samples of 502ys and Nipponbare were harvested from four-week-old seedlings grown in natural conditions. Leaf sections were fixed in a solution of $3 \%$ glutaraldehyde and post-fixed with $1 \%$ osmium tetroxide. The tissues were dehydrated in a gradient acetone series and embedded in Epon812 medium prior to thin sectioning. The samples were stained with uranyl acetate and Reynolds' lead citrate, and observed under a transmission electron microscope (Hitachi H-600IV, Japan).

\section{Analysis of pigments}

Chls were extracted from $0.2 \mathrm{~g}$ fresh leaves of the $502 y \mathrm{~s}$ mutant and its wild-type Nipponbare with $80 \%$ acetone, and Chl contents were determined with UV-1700 UVvisible spectrophotometer (Shimadzu) according to the method of Arnon (1949).

Chls used for HPLC analysis were extracted from fresh rice leaf tissue of four-week-old 502ys and wild-type plants with $100 \%$ acetone. The extract was centrifuged at 7,197 $\mathrm{g}$ (Eppendorf 5430R; 7,830 rpm) for $15 \mathrm{~min}$, and the supernatants were subjected to HPLC on a C18 column (Eclipse XDB-C18, $4.6 \mathrm{~mm}$ i.d. $\times 150 \mathrm{~mm}$ long, $5 \mu \mathrm{m}$; Agilent) and eluted with solvent (methanol: acetonitrile: acetone $=1: 3: 1$ ) at a flow-rate of $1.0 \mathrm{~mL} \mathrm{~min}$ at $40^{\circ} \mathrm{C}$, as previously described (Zapata et al. 2000; Nakanishi et al. 2005; Tanaka et al. 1999, 2010; Zhou et al. 2013). Elution profiles were monitored by measuring $\mathrm{A}_{660}$, and Chl $a$ and $b$ standards (Sigma) were used as control.

\section{Complementation of the 502ys mutant}

For complementation of the 502ys mutation, the fulllength 2,141-bp OsChl P (LOC_OsO2g51080) gene was amplified using primer $1 \mathrm{~F}$ (5' -ACATCTAGAATGACCT CGCTGTCGTCC-3') and primer 1R (5'-AGCCTGCAGTCACAAGGTGACCTTCTC-3') from the wildtype Nipponbare. The primers incorporated an XbaI site at the N-terminal end and a PstI site at the C-terminal end of the gene. The PCR products were inserted into the pMD19-T vector (TaKaRa) and sequenced to obtain the correct clone pMD-OsChl $P$. The pMD-OsChl $P$ plasmid was then digested with XbaI and PstI, and cloned into the corresponding site of the binary vector pCAMBIA2300. The resulting pC2300-OsChl P plasmid, which contained the OsChl $P$ sequence driven by the actin 1 promoter, was introduced into Agrobacterium tumefaciens strain EHA105, and transformed to the 
$502 y s$ mutant for complementation test. To identify whether the wild-type OsChl P gene were inserted into the mutant, the 502ys mutant and its transgenic plants were amplified using primer $2 \mathrm{~F}$ (5'-GAATCCCTCAGCATTGTTC-3') and primer 2R (5'-AGGGCAGCATAAATCAAGT-3') that were designed to anneal to sequences located on the actin 1 promoter and OsChl $P$ gene, respectively, in which the PCR conditions were: $94^{\circ} \mathrm{C}$ for $3 \mathrm{~min} ; 30$ cycles of $94^{\circ} \mathrm{C}$ for $30 \mathrm{~s}, 55^{\circ} \mathrm{C}$ for $30 \mathrm{~s}$, $72^{\circ} \mathrm{C}$ for $1 \mathrm{~min}$; and finally $72^{\circ} \mathrm{C}$ for $7 \mathrm{~min}$.

\section{Results}

\section{Characteristics of the 502ys mutant}

The 502ys mutant was isolated from japonica rice cv Nipponbare via EMS mutagenesis. The mutant exhibited a yellow-green leaf phenotype throughout development and retarded growth rate (Figure 1). Although its days to heading increased $10.4 \mathrm{~d}$, its height of plants and number of productive panicles decreased by $9.8 \%$ and $44.0 \%$ compared with the wild type, respectively (Table 1 ). Besides, its seed-setting rate and 1000-grain weight also declined by $9.1 \%$ and $6.2 \%$, respectively.

To characterize the yellow-green phenotype of the 502ys mutant, we measured its Chl contents, and found its contents of Chl $a, b$, and total Chls to be $28.4 \%$ to $72.8 \%, 17.6 \%$ to $50.7 \%$, and $26.2 \%$ to $67.3 \%$ of those in wild type, respectively (Table 2), which indicated that the mutant phenotype resulted from reduced Chl level.

To explore whether low $\mathrm{Chl}$ content in the mutant leaves affects the chloroplast development and morphology, we examined the ultrastructure of chloroplast using transmission electron microscopy. In wild type, the chloroplasts displayed well-developed membrane systems composed of grana connected by stroma lamellae (Figure 2a and b). However, the grana stacks in the $502 y s$ mutant appeared less dense compared with that in wild type. The thylakoid membrane systems of the mutant chloroplasts were disturbed, and the membrane spacing was not as clear as that in wild-type chloroplasts. In addition, the mutant chloroplasts exhibited vacuolation (Figure $2 \mathrm{c}$ and $\mathrm{d}$ ). These results indicated that the development of chloroplast was suppressed in the 502ys mutant.

\section{Map-based cloning of the mutant gene}

The 502ys mutant was crossed with normal green variety Minghui 63 and wild-type Nipponbare. As result, all $F_{1}$ plants displayed normal green phenotype. $F_{2}$ population from the two crosses showed a segregation ratio of 3:1 (green:yellow-green plants, $\chi^{2}<\chi_{0.05}^{2}=3.84, \mathrm{P}>0.05$ ). The data suggested that the yellow-green phenotype of 502ys mutant is controlled by a single recessive nuclear gene.

To map the 502ys locus, an $\mathrm{F}_{2}$ mapping population was generated from the cross between the 502ys mutant with Minghui 63 (an indica cultivar). The 502ys mutant gene was mapped to an interval between the SSR markers RM318 and RM240 on the long arm of chromosome 2 (Figure 3a). To further define the position of the 502ys gene, six InDel makers were developed (Table 3). As result, the 502ys locus was narrowed down to a $61-\mathrm{kb}$ region between the two InDel makers Y3 and Y4 (Figure 3b).

Within the Y3 and Y4 region, eight putative genes had been annotated in Rice Genome Annotation Project (http://rice.plantbiology.msu.edu). According to recombinants between the two InDel markers and the 502ys mutant gene, we found one gene (LOC_Os02g51080) encoding a putative FAD binding domain containing protein, and sequence BLAST showed its encoded product has a significant similarity to CHL P (73\% identities) in Arabidopsis. Then, we cloned the LOC_Os02g51080 gene from the 502ys mutant and its wild-type, respectively. DNA sequencing results revealed a single nucleotide G-to-A substitution at position 1279 of its coding region in 502ys mutant. Next, its cDNA sequence was

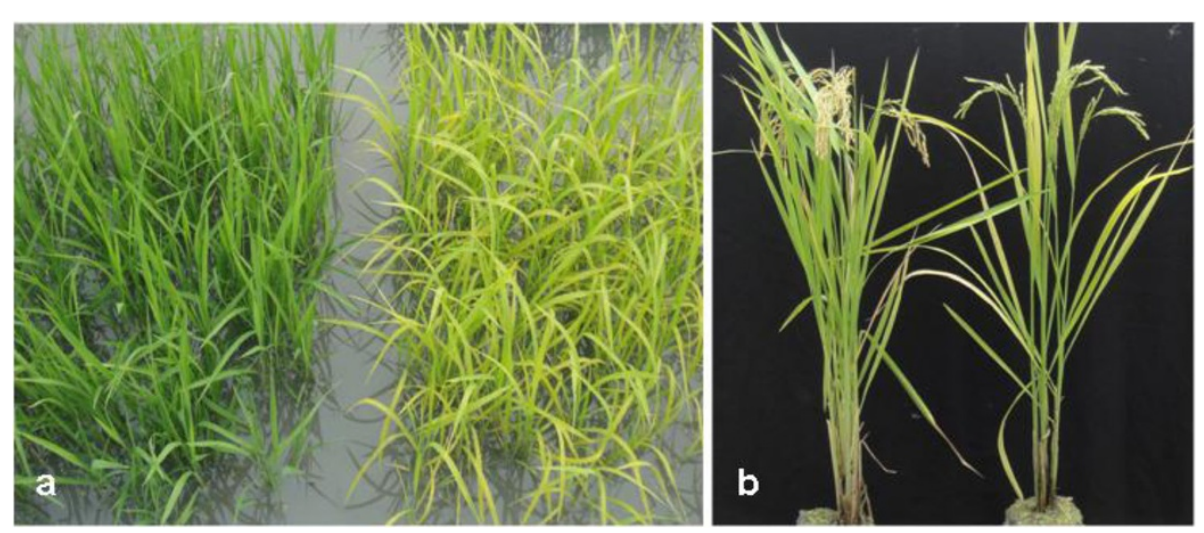

Figure 1 Plant phenotype of the 502ys mutant. a. Four-week-old plants of wild-type Nipponbare (left) and 502ys mutant (right). b. Plants of wild-type Nipponbare (left) and 502ys mutant (right) at mature stage. 
Table 1 Comparison of major agronomic traits between the 502ys mutant and its wild-type Nipponbare

\begin{tabular}{lccc}
\hline Traits & Nipponbare (WT) & 502ys & Compared with WT (\%) \\
\hline Days to heading (d) & $87.3 \pm 0.6$ & $97.7 \pm 2.5$ & $+11.9 \%^{*}$ \\
Plant height (cm) & $104.0 \pm 2.4$ & $93.8 \pm 1.6$ & $-9.8 \%^{*}$ \\
No. of productive panicles per plant & $10.9 \pm 0.7$ & $6.1 \pm 0.6$ & $-44.0 \%^{*}$ \\
No. of spikelets per panicle & $116.3 \pm 3.2$ & $113.1 \pm 2.4$ & $-2.8 \%$ \\
Seed-setting rate (\%) & $87.4 \pm 2.5$ & $78.3 \pm 1.9$ & $-9.1 \%^{*}$ \\
1000-grain weight (g) & $27.4 \pm 0.3$ & $25.7 \pm 0.4$ & $-6.2 \%^{*}$ \\
\hline
\end{tabular}

"Significantly different at $P=0.05$.

amplified by reverse transcription (RT)-PCR, and the G-to-A substitution at codon 616 (corresponding to position 1279 in the coding region) was confirmed in the mutant, which resulted in an amino acid change from Gly-206 to Ser (Figure 3d) in the encoded protein. Thus, LOC_Os02g51080 gene was considered as the candidate gene of 502ys, and designated tentatively as OsChl P gene. In addition, we also sequenced the 1975-bp upsteam of $L O C \_O s 02 g 51080$ gene and found its promoter region did not change in the 502ys mutant.

\section{Characterization of the $\mathrm{OsChl} P$ gene}

Sequence comparison between genomic DNA and cDNA revealed that the OsChl $P$ gene is comprised of three exons and two introns. The cDNA sequence of OsChl P consists of $1392 \mathrm{bp}$ and encodes a 463-amino acid protein with a molecular mass of approximately $50 \mathrm{kD}$. OsCHL $\mathrm{P}$ protein contains an apparent chloroplast-targeting sequence of 37 amino acid residues at its $\mathrm{N}$ terminus (http://www. cbs.dtu.dk/services/TargetP/; Additional file 1: Figure S1).

BlastP search indicated that CHL P exists widely in various species, including higher plant, moss, Chlorophyceae and Cyanobacteria. Multiple amino acid sequence alignment suggested that OsCHL $\mathrm{P}$ has a high similarity to monocotyledonous plant sorghum (Sorghum bicolor), maize (Zea mays) and wheat (Triticum aestivum) CHL P, and dicotyledons plant cucumer (Cucumis sativus) and Arabidopsis CHL P, with identity of 93\%, 92\%, 91\%, $81 \%$ and $73 \%$, respectively. In addition, OsCHL $\mathrm{P}$ is also $77 \%$ and $76 \%$ identical to $\mathrm{CHL} \mathrm{P}$ proteins in fruiter species, olive and peach, respectively (Additional file 1: Figure S1). At the same time, we analyzed the possible phylogenetic relationships between OsCHL P and its related proteins from higher plants and other photosynthetic organisms, indicating that the OsCHL P is more closely related to $\mathrm{CHL} P$ proteins from the monocotyledonous plants sorghum, maize and wheat than to those of other species (Figure 4).

\section{Analysis of chlorophylls}

Because CHL P catalyzes the reduction of $\mathrm{Chl}_{\mathrm{GG}}$ to $\mathrm{Chl}_{\text {Phy }}$ (Keller et al. 1998), the final product of Chl biosynthesis should be $\mathrm{Chl}_{\mathrm{GG}}$ when the plants have a defect in CHL P. So we examined Chl compositions of the $502 y s$ mutant by HPLC. The result showed that the majority of Chl molecules in the mutant were conjugated with an unsaturated geranylgeraniol side chain, in addition to small amount of normal $\mathrm{Chl}_{\text {Phy }}$ (Figure 5b; Tanaka et al. 2010; Zhou et al. 2013), indicated that the mutant has a defective OsCHL P. Therefore, we concluded the candidate gene underlying the 502ys mutant phenotype is OsChl P.

\section{Complementation analysis}

The identity of OsChl P was subsequently confirmed by genetic complementation experiments. The plasmid pC2300-OsChl P, containing the entire OsChl P ( $L O C \_O$ s02g51080) genomic sequence of the wild-type Nipponbare under the control of the actin 1 promoter, was introduced into the 502ys mutant, and 87 independent transgenic lines were obtained. Among them, 78 PCRpositive lines showed a complementation of the $502 y \mathrm{~s}$ phenotype and their growth rates were nearly identical to those of wild-type plants, whereas the other 9 PCR-negative lines failed to rescue the 502ys mutant

Table 2 Pigment contents in leaves of the 502ys mutant and its wild-type Nipponbare, in $\mathrm{mg} \mathrm{g}_{\text {fresh weight }}^{-1}$

\begin{tabular}{|c|c|c|c|c|c|}
\hline Growth stage & Line & Total Chl & $\mathrm{Chl} a^{\mathrm{a}}$ & Chl $b^{\mathrm{a}}$ & Chl a/b Ratio \\
\hline \multirow[t]{2}{*}{ Seedling stage } & Wild-type & $2.84 \pm 0.19$ & $2.13 \pm 0.10$ & $0.71 \pm 0.09$ & $3.00 \pm 0.14$ \\
\hline & $502 y s$ & $1.91 \pm 0.08$ & $1.55 \pm 0.09$ & $0.36 \pm 0.03$ & $4.31 \pm 0.32$ \\
\hline \multirow[t]{2}{*}{ Heading stage } & Wild-type & $3.36 \pm 0.03$ & $2.68 \pm 0.02$ & $0.68 \pm 0.06$ & $3.94 \pm 0.22$ \\
\hline & $502 y s$ & $0.88 \pm 0.04$ & $0.76 \pm 0.02$ & $0.12 \pm 0.02$ & $6.33 \pm 0.48$ \\
\hline
\end{tabular}

${ }^{\mathrm{a}}$ Chls were measured in $80 \%$ acetone extracts from the first, second and third leaves from the top at indicated growth stages. Values shown are the mean \pm SD from five independent determinations. 

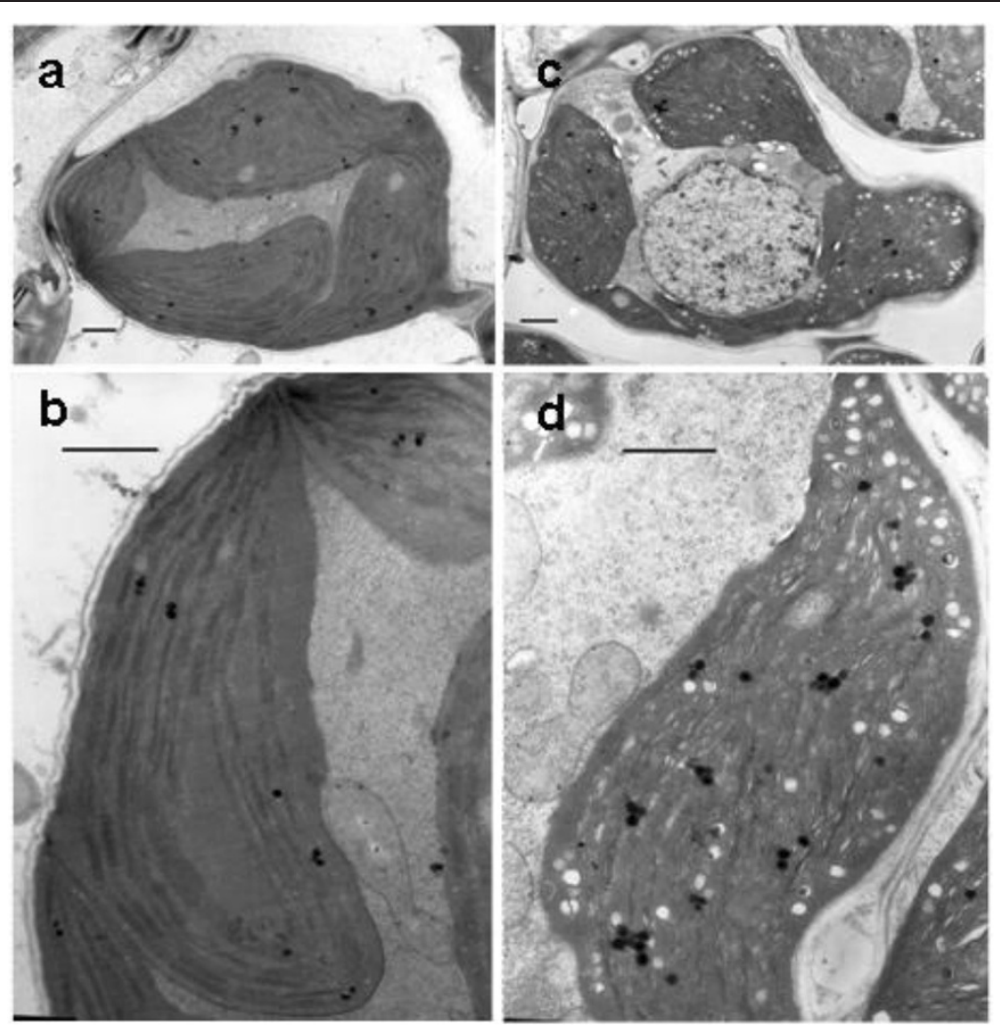

Figure 2 Electron microscopic analysis of the rice 502ys mutant. a. and $\mathbf{b}$. Mesophyll cells and chloroplast of wild-type Nipponbare, respectively. c. and d. Mesophyll cell and chloroplast of 502ys mutant, respectively. Bar equals $1 \mu \mathrm{m}$.

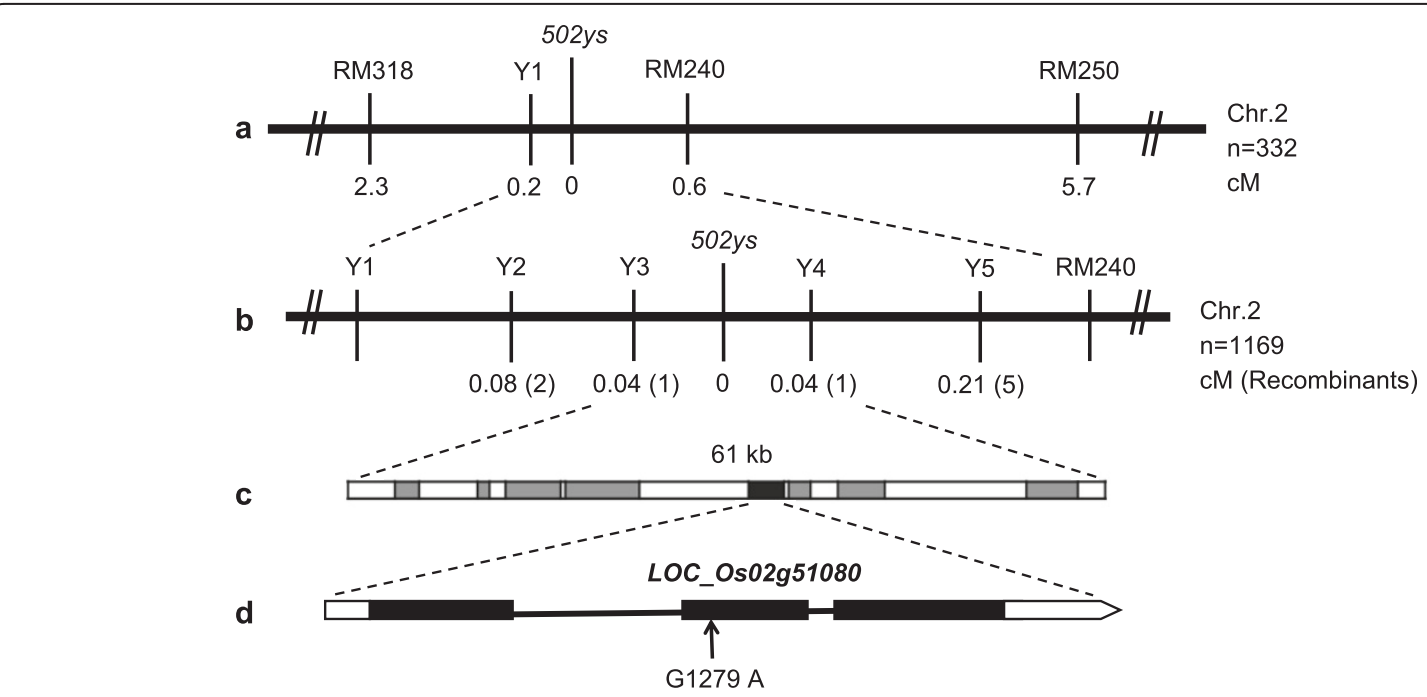

Figure 3 Fine mapping of the 502ys locus. al. The 502ys locus was mapped to a region between InDel marker Y1 and SSR marker RM240 on the long arm of rice chromosome 2. b. The 502ys locus was finely mapped to a region between InDel markers Y3 and Y4. c. The 61-kb region contains eight putative genes. $\mathbf{d}$. The candidate LOC_Os02g51080 gene is comprised of three exons and two introns, and a single nucleotide G-to-A substitution at position 1279 of its coding region was found in the 502ys mutant. 
Table 3 Insertion/deletion (InDel) makers used for fine mapping of the 502ys locus

\begin{tabular}{|c|c|c|}
\hline Maker & Forward primer $\left(5^{\prime}-3^{\prime}\right)$ & Reverse primer $\left(5^{\prime}-3^{\prime}\right)$ \\
\hline $\mathrm{Y1}$ & GAAGATGATGCCAACTGA & GAGCCAATCCACAATACA \\
\hline Y2 & ССTCCTTAACTITGCCTCTG & AGCCAGTAATAGCGTCCTCA \\
\hline Y3 & CCGTGGTTCAGTCAAAGA & AACGCGGTGATACATTCC \\
\hline Y4 & TATGGACGAGATGAGGAG & TAAGAGGCAGGTTGAAAA \\
\hline Y5 & AGAGGCGAGGAGGATTGG & AGGGCAGTAGCAGTGCAAGA \\
\hline
\end{tabular}

(Figure 6). Furthermore, HPLC analyses confirmed that the PCR-positive transgenic lines containing the wild-type OsChl $P$ gene all accumulated normal $\mathrm{Chl}_{\text {phy }}$ (Figure $5 \mathrm{C}$ ). Therefore, we concluded that we succeeded to clone $C h l$ $P$ gene in rice, and that the 502ys mutant resulted from a single base pair mutation in OsChl P gene.

\section{Discussion}

CHL P reduces GGPP to PhyPP and provides the side chain for $\mathrm{Chl}$, tocopherols, and plastoquinones, which is prerequisite to their integration into plastid membranes (Soll et al. 1980, 1983; Soll 1987; Bollivar et al. 1994; Graßes et al. 2001; Giannino et al. 2004). Addlesee et al. (1996) cloned the chlP gene from the cyanobacterium Syneclrocystis sp. PCC 6803, and subsequently demonstrated this cyanobacterium gene was able to partially complement a bchP mutant of purple photosynthetic bacterium $R$. sphaeroides. Addlesee and Hunter (1999) identified the bchP gene in Rhodobacter sphaeroides transposon mutant, and demonstrated the activity of a $b c h P$ gene product using the recombinant protein. Keller et al. (1998) cloned a cDNA encoding CHL P from Arabidopsis thaliana and suggested that a multifunctional CHL P catalyzed the stepwise reduction of $\mathrm{Chl}_{\mathrm{GG}} a$ into $\mathrm{Chl}_{\mathrm{phy}} a$ as well as the reduction of GGPP into phyPP. Tanaka et al. (1999) cloned a tobacco cDNA sequence encoding CHL $\mathrm{P}$, and showed reduction in Chl and tocopherol contents and accumulation of partially $\mathrm{Chl}_{\mathrm{GG}}$ in transgenic tobacco expressing antisense Chl $P$. Giannino et al. (2004) identified a Chl $P$ gene in peach and suggested that its expression was regulated by photosynthetic activity. Bruno et al. (2009) characterized a Chl $P$ gene in olive, and found that OeChl $P$ expression was modulated during leaf and fruit development and rapidly enhanced under stress conditions. Park et al. (2010) isolated a Chl P cDNA in sesame and showed SiChl $P$ expression was induced by light but repressed by dark, ABA and ethylene. However, no Chl P gene had been identified in monocotyledonous plants until 2012. Most recently, Zhou et al. (2013) isolated a yellow leaf mutant (lyl1) from the progeny of a japonica rice $\mathrm{ZH} 11$ treated with ${ }^{60} \mathrm{Co}$ radiation, and demonstrated the $l y l 1$ mutant phenotype was caused by a point mutation in the LOC_Os02g51080 gene for CHL P via map-based cloning and RNA interference (RNAi) approaches. In present study, we isolated the yellow-green leaf mutant $502 y$ s from a japonica rice Nipponbare through EMS mutagenesis and cloned OsChl $P$ gene ( $L O C \_O s O 2 g$ 51080). In the 502ys mutant, DNA sequence of the OsChl $P$ gene had a single base pair mutation, resulting in an amino acid change in the encoded protein, and the majority of Chl molecules were conjugated with an unsaturated geranylgeraniol side chain. Furthermore, the mutant phenotype was rescued by transformation with

ᄂ 0.05

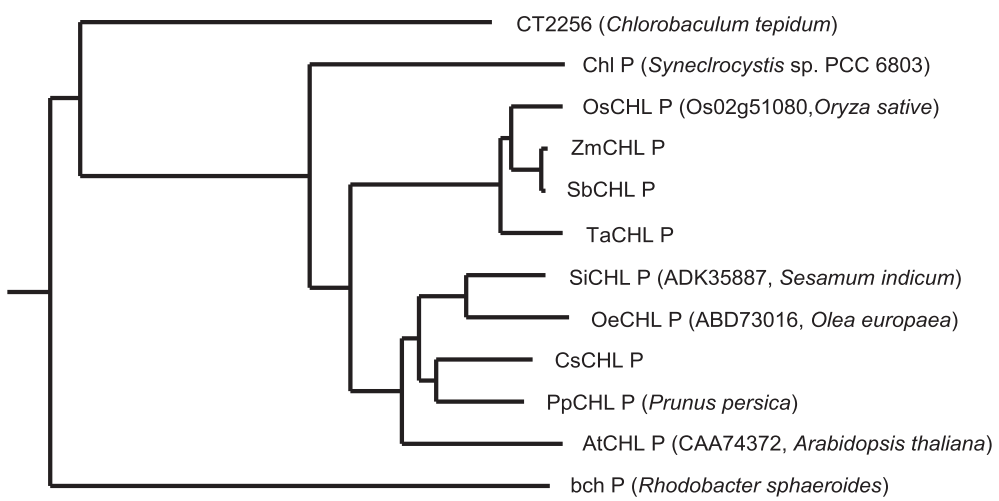

Figure 4 Phylogenetic tree representing alignment of OsCHL P protein and its homologues. The rooted neighbor-joining tree using percentage identities was constructed based on a multiple sequence alignment generated with the program DNAMAN. Accession numbers for the respective protein sequences are as follows: Oryza sativa (OsCHL P, LOC_Os02g51080), Sorghum bicolor (SbCHL P, XP_002454287), Zea mays (ZmCHL P, NP_001149382), Triticum aestivum (TaCHL P, AAZ67145), Arabidopsis thaliana (AtCHL P, CAA74372), Cucumis sativus (CsCHL P, XP_004138167), Prunus persica (PpCHL P, EMJ10306), Sesamum indicum (SiCHL P, ADK35887), Olea europaea (OeCHL P, ABD73016), Synechocystis sp. PCC 6803 (Chl P, NP_441659), Chlorobium tepidum TLS (CT2225, AAM73471), Rhodobacter sphaeroides (bch P, AAF24283). Scale represents percentage substitution per site. 


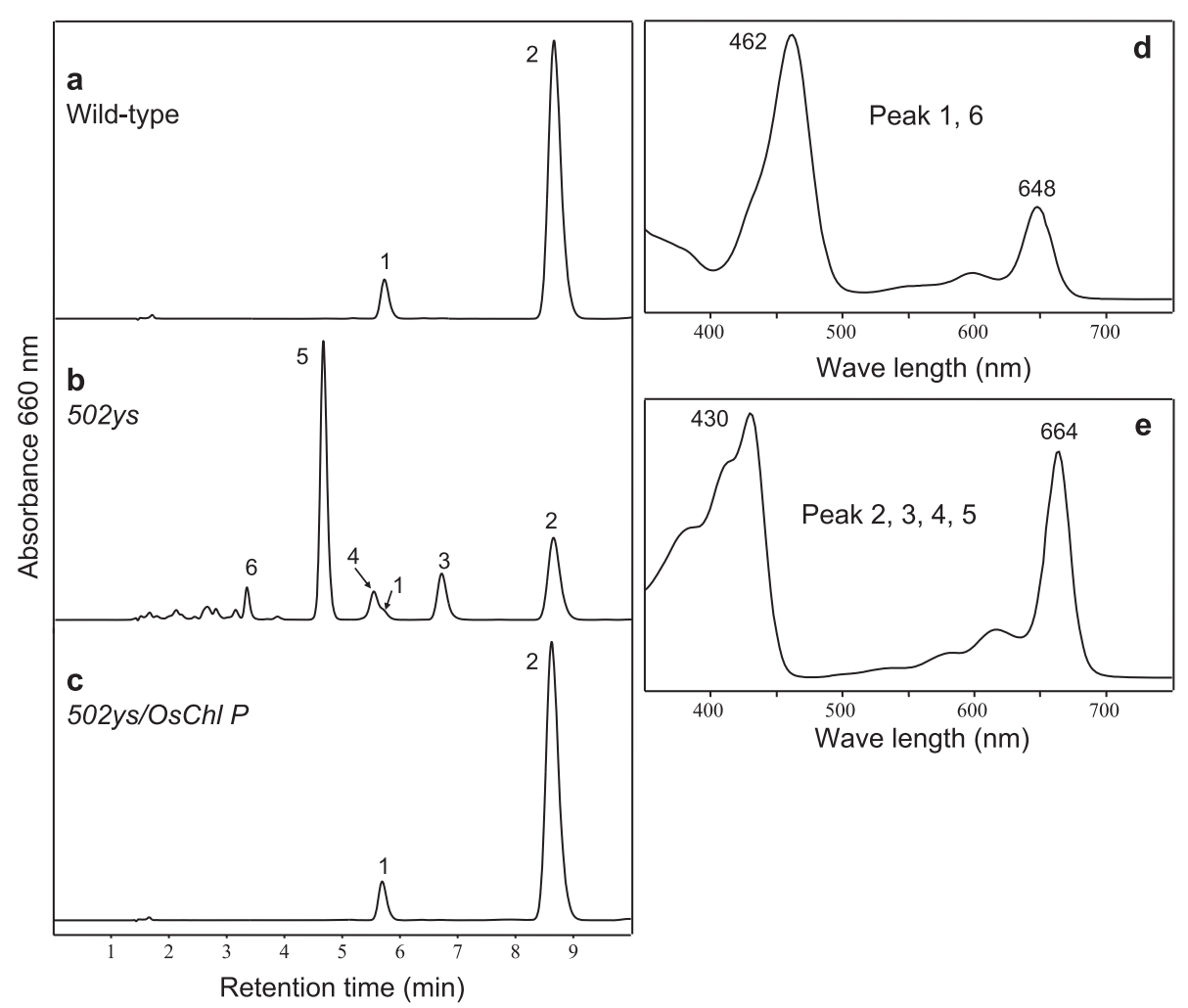

Figure 5 HPLC analysis of Chls. The elution profiles of wild type (a), 502ys mutant (b) and 502ys/OsCHL $P$ transgenic line (c) were detected at $660 \mathrm{~nm}$. Peak 1, Chl b; peak 2, Chl $a$; peak 3, 4 and 5, Chl $\mathrm{THGG}_{1} a, \mathrm{Chl}_{\mathrm{DHGG}} a$ and $\mathrm{Chl}_{\mathrm{GG}} a$, respectively; peak 6, Chl $\mathrm{GG} b$. The absorption spectra of the peaks 1 and 6 (d) and the peaks 2, 3, 4 and 5 (e) in acetone were compared, respectively.

the wild-type gene. Therefore, a Chl P gene was identified successfully in monocotyledonous plants.

Several Chl P (bchP) deficient mutants have been identified. A $b c h P$ mutant of the purple photosynthetic bacterium Rhodobacter sphaeroides accumulated only $\mathrm{bChl}_{\mathrm{GG}}$ and the maximal photosynthetic growth rate were significantly reduced (Addlesee et al. 1996; Addlesee and Hunter 1999). Transgenic tobacco expressing antisense Chl $P$ exhibited reduction in $\mathrm{Chl}$ and tocopherol contents, and up to $58 \%$ of Chls was esterified with geranylgeraniol instead of phytol under low light conditions (Tanaka et al. 1999). In addition, the transgenic plants were much more sensitive to light stress (Graßes et al. 2001). Graßes et al. (2001) suggested that $\mathrm{Chl}_{\mathrm{GG}}$ did not affect the harvesting and transfer of light energy, but the reduced tocopherol content is a limiting factor for defensive reaction to photo-oxidative stress of the transgenic plants. In this study, the 502ys mutant exhibited a yellow-green leaf
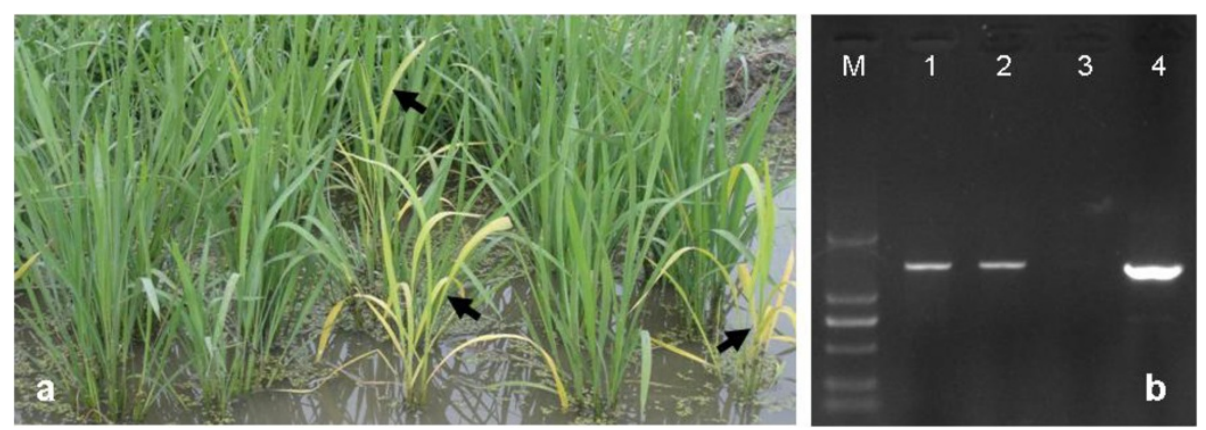

Figure 6 Complementation of the 502ys mutant by the wild-type OsChI P gene. a. Phenotype of $T_{0}$ transgenic plants. Arrowheads indicated PCR-negative transgenic plants, the others plants were PCR-positive transgenic plants. b. Identification of transgenic plants by PCR. M, DL-2000 marker; 1 and 2, PCR-positive transgenic plants; 3, The 502ys mutant (PCR-negative control) or PCR-negative transgenic plants; 4, pC2300-OsChI P plasmid (control). 
phenotype, reduced Chl level, arrested chloroplast development, and retarded growth rate. HPLC analysis of Chls indicated that the 502ys mutant accumulated Chls esterified with phytol, GG, dihydro-GG, and tetrahydro-GG. Our data are consistent with the above-mentioned reports that the Chl P mutants were capable of photosynthetic growth using Chls with an unsaturated geranylgeraniol side chain, although their growth rate was decreased remarkably.

Arabidopsis CHL P was able to catalyze the reduction of $\mathrm{Chl}_{\mathrm{GG}} a$ into $\mathrm{Chl}_{\mathrm{Phy}} a$ (Keller et al. 1998). The 502ys mutant accumulated $\mathrm{Chl}_{\mathrm{GG}} a, \mathrm{Chl}_{\mathrm{DHGG}} a, \mathrm{Chl}_{\mathrm{THGG}} a$ and $\mathrm{Chl}_{\text {Phy }} a$, which implied that OsCHL $\mathrm{P}$ is also involved in the reduction of $\mathrm{Ch}_{\mathrm{GG}} a$. However, when the recombinant OsCHL P protein was expressed in E. coli, we found that only a very small amounts of $\mathrm{Chl}_{\mathrm{GG}} a$ was detected to be converted to the $\mathrm{Chl}_{\mathrm{DHGG}} a$ after $5 \mathrm{~h}$ of incubation with the recombinant protein (data not show), implying its enzymatic activity were very low when $\mathrm{Chl}_{\mathrm{GG}} a$ was used as substrate. It is possible that the recombinant $\mathrm{OsChl} P$ product did not form a proper conformation, or the enzyme reaction system was not optimal, or additional proteins were required for its catalytic activity. In any case, further studies are necessary to clarify the function of the OsCHL P protein.

\section{Additional file}

Additional file 1: Figure S1. Alignment of the deduced amino acid sequence of OsCHL P and its homologues. Identical residues were boxed in black, similar residues ( $\geq 75 \%$ identical) were highlighted in gray. The red arrowhead indicates mutational site (G206S) of the 502ys mutant, and the red underline indicates the putative chloroplast-targeting sequence of 37 amino acid residues at its $\mathrm{N}$ terminus. Accession numbers for the respective protein sequences are as Figure 4 .

\section{Abbreviations}

Chl: Chlorophyll; CHL P: Geranylgeranyl reductase; Chl phy: Phytyl chlorophyll; $\mathrm{Ch}_{\mathrm{GG}}$ : Geranylgeranylated chlorophyll; Chl $\mathrm{DHGG}$ : Dihydrogeranylgeranyl

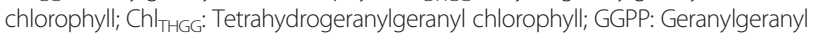
diphosphate; PhyPP: Phytyl pyrophosphate.

\section{Competing interests}

The authors declare that they have no competing interests.

\section{Author's contributions}

PYW carried out most of the experiments and drafted the manuscript. CML, YW and RH participated in function analysis of the transgenic plants. CHS, ZJX, JQZ and XLG provided technical assistance. XJD provided the mutant material, designed the experimental plan and revised the manuscript. PRW designed the experimental plan, participated in part of the experiments, analyzed the data, drafted and revised the manuscript. All authors read and approved the final manuscript.

\section{Acknowledgments}

Financial support from the National Natural Science Foundation of China (31171533, 31071402, and 91335107). We thank Prof. Chengcai Chu from Institute of Genetics and Developmental Biology, Chinese Academy of Sciences, for kindly providing the binary vector pCAMBIA2300.
Received: 10 April 2014 Accepted: 14 April 2014

Published: 24 April 2014

\section{References}

Addlesee HA, Hunter CN (1999) Physical mapping and functional assignment of the geranylgeranyl-bacteriochlorophyll reductase gene, bchP, of Rhodobacter sphaeroides. J Bacteriol 181:7248-7255

Addlesee HA, Gibson LCD, Jensen PE, Hunter CN (1996) Cloning, sequencing and functional assignment of the chlorophyll biosynthesis gene, chZP, of Synechocystis sp. PCC6803. FEBS Lett 389:126-130

Arnon DI (1949) Copper enzymes in isolated chloroplasts: polyphenoloxidase in Beta vulgaris. Plant Physiol 24:1-15

Beale SI (2005) Green genes gleaned. Trends Plant Sci 10:309-312

Bollivar DW, Wang SJ, Allen JP, Bauer CE (1994) Molecular genetic analysis of terminal steps in bacteriochlorophyll a biosynthesis: characterization of a Rhodobacter capsulatus strain that synthesizes geranylgeraniol-esterified bacteriochlorophyll $a$. Biochemistry 33:12763-12768

Bruno L, Chiappetta A, Muzzalupo I, Gagliardi C, laria D, Bruno A, Greco M, Giannino D, Perri E, Bitonti MB (2009) Role of geranylgeranyl reductase gene in organ development and stress response in olive (Olea europaea) plants. Funct Plant Biol 36:370-381

Cahoon EB, Hall SE, Ripp KG, Ganzke TS, Hitz WD, Coughlan SJ (2003) Metabolic redesign of vitamin $E$ biosynthesis in plants for tocotrienol production and increased antioxidant content. Nat Biotechnol 21:1082-1087

Chew AGM, Frigaard NU, Bryant DA (2008) Identification of the bchP Gene, encoding geranylgeranyl reductase in Chlorobaculum tepidum. J Bacteriol 190:747-749

Collakova E, DellaPenna D (2001) Isolation and functional analysis of homogentisate phytyltransferase from Synechocystis sp. PCC6803 and Arabidopsis. Plant Physiol 127:1113-1124

Emanuelsson O, Nielsen H, Brunak S, Heijne GV (2000) Predicting subcellular localization of protein based on their $\mathrm{N}$-terminal amino acid sequence. J Mol Biol 300:1005-1016

Giannino D, Condello E, Bruno L, Testone G, Tartarini A, Cozza R, Innocenti AM, Bitonti MB, Mariotti D (2004) The gene geranylgeranyl reductase of peach (Prunus persica [L.] Batsch) is regulated during leaf development and responds differentially to distinct stress factors. J Exp Bot 55:2063-2073

Graßes T, Grimm B, Koroleva O, Jahns P (2001) Loss of a-tocopherol in tobacco plants with decreased geranylgeranyl reductase activity does not modify photosynthesis in optimal growth conditions but increases sensitivity to high-light stress. Planta 213:620-628

Islam MR, Aikawa S, Midorikawa T, Kashino Y, Satoh K, Koike H (2008) slr1923 of Synechocystis sp. PCC6803 is essential for conversion of 3,8-divinyl(proto) chlorophyll(ide) to 3-monovinyl(proto)chlorophyll(ide). Plant Physiol 148:1068-1081

Keller Y, Bouvier F, d'Harlingue A, Camara B (1998) Metabolic compartmentation of plastid prenyllipid biosynthesis: evidence for the involvement of a multifunctional CHLP. Eur J Biochem 251:413-417

Lichtentaler HK, Schwender J, Disch A, Rohmer M (1997) Biosynthesis of isoprenoids in higher plant chloroplast proceeds via a mevalonateindependent pathway. FEBS Lett 400:271-274

McCouch SR, Teytelman L, Xu YB, Lobos KB, Clare K, Walton M, Fu BY, Maghirang R, Li ZK, Xing YZ, Zhang QF, Kono I, Yano M, Fjellstrom R, DeClerck G, Schneider D, Cartinhour S, Ware D, Stein L (2002) Development and mapping of 2240 new SSR markers for rice (Oryza sativa L.). DNA Res 9:199-207

Nagata N, Tanaka R, Satoh S, Tanaka A (2005) Identification of a vinyl reductase gene for chlorophyll synthesis in Arabidopsis thaliana and implications for the evolution of Prochlorococcus species. Plant Cell 17:233-240

Nakanishi H, Nozue H, Suzuki K, Kaneko Y, Taguchi G, Hayashida N (2005) Characterization of the Arabidopsis thaliana mutant $p c b 2$ which accumulates divinyl chlorophylls. Plant Cell Physiol 46:467-473

Park MR, Cho EA, Rehman S, Yun SJ (2010) Expression of a sesame geranylgeranyl reductase $C D N A$ is induced by light but repressed by abscisic acid and ethylene. Pak J Bot 42:1815-1825

Rohmer M, Knani M, Simonin P, Sutter B, Sahm H (1993) Isoprenoid biosynthesis in bacteria: a novel pathway for the earlysteps leading to isopentenyl diphosphate. Biochem J 295:517-524

Sakuraba Y, Rahman ML, Cho SH, Kim YS, Koh HJ, Yoo SC, Peak NC (2013) The rice faded green leaf locus encodes protochlorophyllide oxidoreductase B 
and is essential for chlorophyll synthesis under high light conditions. Plant J 74:122-133

Savidge B, Weiss JD, Wong YH, Lassner MW, Mitsky TA, Shewmaker CK, Post-Beittenmiller D, Valentin HE (2002) Isolation and characterization of homogentisate phytyltransferase genes from Synechocystis sp. PCC6803 and Arabidopsis. Plant Physiol 129:321-332

Schultz G, Soll J, Fiedler E, Schulze-Siebert D (1985) Synthesis of prenylquinones in chloroplasts. Physiol Plant 64:123-129

Soll J (1987) a-Tocopherol and plastoquinone synthesis in chloroplast membranes. Methods Enzymol 148:383-392

Soll J, Kemmerling M, Schultz G (1980) Tocopherol and plastoquinone synthesis in spinach chloroplasts subfractions. Arch Biochem Biophys 200:544-550

Soll J, Schultz G, Rüdiger W, Benz J (1983) Hydrogenation of geranylgeraniol: two pathways exist in spinach chloroplast. Plant Physiol 71:849-854

Tanaka R, Oster U, Kruse E, Rudiger W, Grimm B (1999) Reduced activity ofgeranylgeranyl reductase leads to loss of chlorophyll and tocopherol and to partially geranylgeranylated chlorophyll in transgenic tobacco plants expressing antisense RNA for geranylgeranyl reductase. Plant Physiol 120:695-704

Tanaka R, Rothbart M, Oka S, Takabayashi A, Takahashi K, Shibata M, Myouga F, Motohashi R, Shinozaki K, Grimm B, Tanaka A (2010) LIL3, a light-harvesting-like protein, plays an essential role in chlorophyll and tocopherol biosynthesis. Proc Natl Acad Sci U S A 107:16721-16725

Wang PR, Gao JX, Wan CM, Zhang FT, Xu ZJ, Huang XQ, Sun XQ, Deng XJ (2010) Divinyl chlorophyll $a$ can be converted to monovinyl chlorophyll $a$ by a divinyl reductase in rice. Plant Physiol 153:994-1003

Wang PR, Wan CM, Xu ZJ, Wang PY, Wang WM, Sun CH, Ma XZ, Xiao YH, Zhu JQ, Gao XL, Deng XJ (2013) One divinyl reductase reduces the 8-vinyl groups in various intermediates of chlorophyll biosynthesis in a given higher plant species, but the isozyme differs between species. Plant Physiol 161:521-534

Wu ZM, Zhang X, He B, Diao LP, Sheng SL, Wang JL, Guo XP, Su N, Wang LF, Jiang L, Wang CM, Zhai HQ, Wan JM (2007) A chlorophyll-deficient rice mutant with impaired chlorophyllide esterification in chlorophyll biosynthesis. Plant Physiol 145:29-40

Zapata M, Rodríguez F, Garrido JL (2000) Separation of chlorophylls and carotenoids from marine phytoplankton: a new HPLC method using a reversed phase C8 column and pyridine containing mobile phases. Mar Ecol Prog Ser 195:29-45

Zhou Y, Gong ZY, Yang ZF, Yuan Y, Zhu JY, Wang M, Yuan FH, Wu SJ, Wang ZQ, Yi CD, Xu TH, Ryom MC, Gu MH, Liang GH (2013) Mutation of the lightinduced yellow leaf 1 gene, which encodes a geranylgeranyl reductase, affects chlorophyll biosynthesis and light sensitivity in rice. PLoS ONE 8:e75299

doi:10.1186/2193-1801-3-201

Cite this article as: Wang et al:: Identification of a Geranylgeranyl reductase gene for chlorophyll synthesis in rice. SpringerPlus 2014 3:201.

\section{Submit your manuscript to a SpringerOpen ${ }^{\circ}$ journal and benefit from:}

- Convenient online submission

- Rigorous peer review

- Immediate publication on acceptance

- Open access: articles freely available online

- High visibility within the field

- Retaining the copyright to your article

Submit your next manuscript at $>$ springeropen.com 